livraisons

d'Histoire

de l'Architecture

\section{Livraisons de l'histoire de l'architecture}

$40 \mid 2020$

À propos des princes de Conti

\title{
Les hôtels des princes de Conti à Paris (1660-1751)
}

Les hôtels des princes de Conti à Paris (1660-1751)

The Princes of Conti's Townhouses in Paris (1660-1751)

Die Hôtels der Fürsten von Conti in Paris (1660-1751)

\section{Étienne Faisant}

\section{OpenEdition}

Journals

Édition électronique

URL : http://journals.openedition.org//ha/1252

DOI : $10.4000 /$ lha. 1252

ISSN : 1960-5994

\section{Éditeur}

Association Livraisons d'histoire de l'architecture - LHA

Édition imprimée

Date de publication : 3 décembre 2020

Pagination : 11-22

ISSN : 1627-4970

Référence électronique

Étienne Faisant, «Les hôtels des princes de Conti à Paris (1660-1751) », Livraisons de I'histoire de l'architecture [En ligne], 40 | 2020, mis en ligne le 28 décembre 2020, consulté le 24 mars 2021. URL : http://journals.openedition.org//ha/1252 ; DOI : https://doi.org/10.4000//ha.1252 


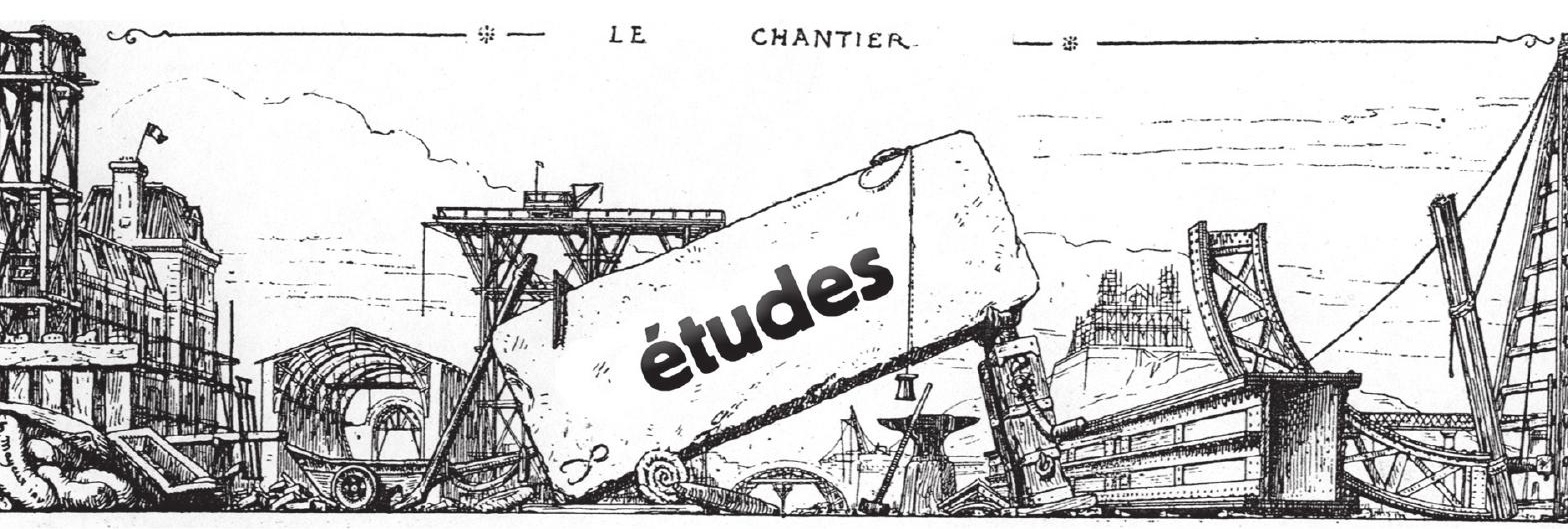





\section{Par Étienne FAISANT}

\section{LES HÔTELS DES PRINCES DE CONTI À PARIS (1660-1751)}

L'acquisition par le fondateur de la seconde maison de Conti d'un hôtel à Paris est une conséquence, très secondaire mais inattendue, de la paix des Pyrénées. Armand de Bourbon (1629-1666), fils d'Henri II, prince de Condé, a en effet longtemps habité l'hôtel de ses parents, à Saint-Germain-des-Prés : si son frère aîné vécut dès le début des années 1640 dans sa propre demeure, qui paradoxalement était celle dont son cadet avait la jouissance en tant qu'abbé de Saint-Denis ${ }^{1}$, le jeune prince de Conti se réinstalla, après ses années au collège de Clermont ${ }^{2}$, chez sa mère ${ }^{3}$. Après la mort de celle-ci, la propriété de l'hôtel de Condé revint en 1651 à l'aîné, mais les deux frères y habitèrent ensemble jusqu'à ce que leur implication dans la Fronde et l'avancée des troupes royales ne les forcent à fuir Paris ${ }^{4}$. Le Grand Condé poursuivit alors la lutte, ce qui entraîna la saisie de ses biens, mais le prince de Conti se rallia pour sa part au roi en 1653 et entreprit de mettre la main sur une part de la fortune de son frère ${ }^{5}$. Il entra ainsi en possession en septembre 1654 de l'hôtel de Condé, qu'il habita dès lors durant ses séjours à Paris ${ }^{6}$. On comprend donc que lorsque, le 7 novembre 1659, la signature de la paix entrâna le pardon et le prochain retour du prince rebelle, Armand de Bourbon n'ait pas attendu l'arrivée de son aîné pour évacuer cette demeure qu'il occupait alors qu'elle ne lui appartenait pas : dès le 9 janvier 1660, il loua une maison où il fit transporter ses meubles et où il logea ses domestiques, tandis que lui-même s'installait dans

1. Le prince de Condé fit faire des travaux dans l'hôtel de Saint-Denis rue des Haudriettes en 1642 1643 (Min. centr., ét. XCII, 114, 8 novembre 1642 et 115, 4 janvier et 16 février 1643). Le premier devis indique que ces transformations étaient "pour monseigneur le prince de Conty ", mais le dernier concerne les aménagements nécessaires "pour loger les suisses de monseigneur le duc d'Anguien ", et c'est bien ce dernier qui y logeait ensuite (Arch. nat., Min. centr., ét. XCII, 115, 28 mars 1643).

2. Arch. nat., Min. centr., ét. XCII, 119, 11 février 1644.

3. Il habita plus précisément le rez-de-chaussée du pavillon que sa mère avait fait bâtir par François Mansart (Arch. nat., $Z^{1 \mathrm{~J}}$ 271, 3-15 mars 1651). Sur l'hôtel de Condé, voir Étienne Faisant, "L'hôtel de Condé, une demeure princière au faubourg Saint-Germain (1582-1666) ", Monuments et mémoires de la fondation Eugène Piot, Paris, Académie des Inscriptions et Belles-Lettres, t. 94, 2015, p. 243-283.

4. Arch. nat., Min. centr., ét. XCII, 144, 27 mars et 15 mai 1651.

5. Katia Beguin, "La fortune du Grand Condé: un enjeu de l'après-fronde (1654-1660) ", Revue historique, Paris, Presses universitaires de France, $\mathrm{n}^{\circ}$ 601, janvier-mars 1997, p. 57-84, notamment p. $75-77$.

6. Arch. nat., $\mathrm{R}^{3} 107$, f. $347-349 \mathrm{v}$. 
l'hôtel de la présidente Le Coigneux ${ }^{7}$. Il ne s'agissait toutefois là que d'une solution provisoire, manifestement adoptée dans l'urgence, et le prince de Conti dut se mettre en quête d'un hôtel.

\section{L'hôtel de Conti sur le quai Malaquais}

Armand de Bourbon et son épouse Anne-Marie Martinozzi reçurent alors diverses propositions, "entr'autres celle de l'hostel d'Hémery, à quoy S.A.S. madame avoit beaucoup d'inclination ${ }^{8}$. Nicolas Fouquet était toutefois lui aussi intéressé par cet hôtel, si bien que, averti, le cardinal Mazarin, oncle de la princesse, lui fit savoir "qu'il luy feroit plaisir de ne pas penser à aucune maison jusques à ce que madame la princesse de Conty en eût une ». Le prince fut néanmoins amené à choisir une autre demeure en raison de son manque d'argent comptant: un intermédiaire lui proposa en effet, s'il achetait l'hôtel du comte de Brienne, de lui faire payer les créances qu'il avait sur l'Épargne et même de lui prêter une forte somme. Il apparut ensuite que celle-ci avait été fournie par Fouquet, qui détourna ainsi le couple princier de l'hôtel d'Émery.

Construit en 1630-1632 par le financier Le Barbier' ${ }^{9}$, l'hôtel que les Conti acquirent le 20 septembre 1660 moyennant deux cent mille livres se dressait face à la Seine, sur le quai Malaquais ${ }^{10}$. Il comprenait un corps de logis en fond de cour et, sur la droite de celle-ci, une aile terminée par un gros pavillon qui faisait écho à la maison située de l'autre côté : la volonté de donner ainsi l'apparence d'un ensemble symétrique apparaît clairement sur la gravure que lui a consacrée Jean Marot (ill. 1), qui déforme toutefois assez nettement la réalité pour donner plus de force à cette illusion. Sur le côté droit de la parcelle, derrière l'aile, était par ailleurs ménagé un passage menant à la basse-cour établie le long du jardin qui s'étendait derrière le logis.

Dès qu'il en eut pris possession, le prince de Conti entreprit d'en compléter les dépendances. L'architecte Siméon Lambert commença en effet à travailler à de nouvelles constructions qui furent finalement confiées le 19 décembre à Alexandre Delespine: celui-ci fut chargé d'ajouter trois étages carrés sur les remises de la basse-cour ainsi que sur le petit corps de bâtiment en occupant le fond ${ }^{11}$. Ceci fait, le même entrepreneur s'engagea l'année suivante à bâtir "au bout du jardin qui regarde la fasse de l'hostel de Sad. Altesse " un "bastiment " appliqué " par bas en quatre remises doubles pour mettre deux carrosses » avec, à chaque bout, un escalier

7. Arch. nat., $\mathrm{R}^{3} 108$, f. 539-541v et $\mathrm{R}^{3}$ 108, f. 442-445. L'hôtel Le Coigneux, puis de Villars, est aujourd'hui la mairie du $\mathrm{VII}^{\mathrm{e}}$ arrondissement.

8. Arch. nat., $\mathrm{R}^{3}$ 57, 16 juillet 1675 .

9. Sur cet hôtel avant les princes de Conti, voir Emmanuelle Loizeau, Louis et Clément Métézeau, architectes $d u$ roi, thèse d'histoire de l'art sous la direction de Claude Mignot, Paris-Sorbonne, 2009, t. 2, p. 239-245.

10. Arch. nat., Min. centr., ét. LXV, 52, 20 septembre 1660.

11. Arch. nat., $\mathrm{R}^{3}$ 200, 19 décembre 1660 . 


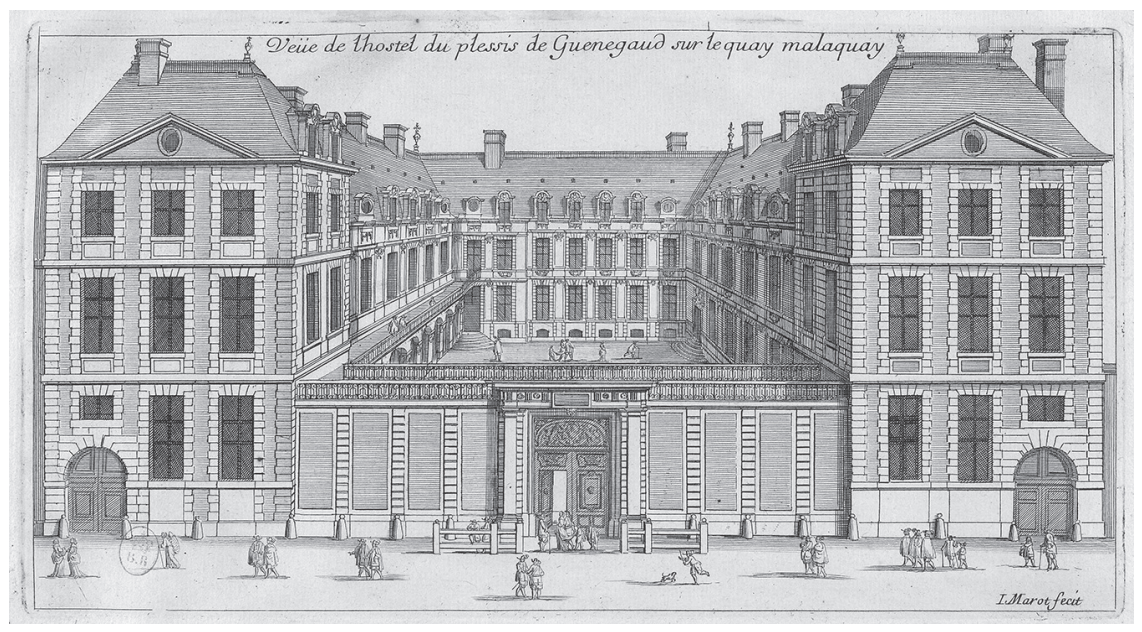

Ill. 1 : "Veue de l'hostel du Plessis de Guénégaud sur le quay Malaquay [ancien hôtel de Conti] ", planche gravée, Jean Marot, Grand Marot, Paris, vers 1670-1679. (C) Cl. BnF/Gallica.

desservant un étage et "des chambres en galletas ${ }^{12}$. Alexandre Delespine promit également à la même occasion au prince de Conti de travailler au "revestement des terres au derrière de son hostel ", ce qui impliquait de construire un mur s'élevant en talus jusqu'à un cordon "de niveau avec les terres du hault ", d'élever au-dessus une balustrade et enfin de lancer au-dessus du vide " un pont pour la communication du corps de logis à la nouvelle court » : on s'employait donc à mettre en place à l'arrière du corps de logis principal une cour anglaise, sans doute destinée à améliorer le sous-sol où était entre autres installée la cuisine. Il faut surtout souligner que le marché désigne l'espace situé derrière l'hôtel comme "la nouvelle court", terme à nouveau explicitement employé en $1670^{13}$ : le prince de Conti avait donc choisi de sacrifier le jardin qui s'était jusqu'alors trouvé là pour lui donner une vocation utilitaire, ce qui explique d'ailleurs que l'on y ait installé des remises à carrosses supplémentaires.

Les travaux menés à l'intérieur même de l'hôtel furent plus réduits, le chantier le plus important, compris dans le même marché de 1661, ayant porté sur le principal escalier de l'hôtel, dont la balustrade fut restaurée et où furent ajoutées des " colomnes d'ordre doricque, tant pour la solidité dudict escallier que pour embellissement d'ycelluy». Le sculpteur Thomas Chenu fournit par ailleurs la même année 1661 un bloc de marbre noir sur lequel était "gravé et doré : l'hostel de Conty ", qui fut installé au-dessus du portail sur le quai ${ }^{14}$. On travailla également

12. Arch. nat., Min. centr., ét. XCV, 704, 3 juin 1661.

13. Arch. nat., Min. centr., ét. XCV, 35, 30 avril 1670 : l'hôtel consistait alors « en plusieurs bastimens, courts sur le devant et sur le derrière". En 1663, les nouvelles remises sont en outre dites " au bout de l'ancien jardin dud. hostel " (Arch. nat., $\mathrm{R}^{3}$ 110, f. 351).

14. Arch. nat., $\mathrm{R}^{3} 108$, f. 455 v. 
à l'aménagement d'une chambre du conseil, où la veuve Capronnier posa « le lambris et armoires " et où Vincent Dulaurier réalisa en 1663 des "ouvrages de peinture et dorure ${ }^{15}$. Les comptes de la maison du prince de Conti énumèrent encore d'autres paiements à des peintres pour des réparations ou de petites interventions dont la nature n'est pas toujours précisée, mais dont le coût modeste témoigne du peu d'importance. Il fut ainsi réalisé pour cinq cents livres d' "ouvrages de peinture " dans l'appartement du prince ${ }^{16}$, mais ce fut là tout ce que l'on jugea nécessaire de faire pour aménager les logements du couple princier. La naissance en 1664 de leur second fils, François-Louis, titré comte de la Marche, ne fut également suivie que par une rapide intervention sur la peinture de "l'alcôve de l'appartement de monseigneur le comte", qui ne fut payée que vingt-cinq livres ${ }^{17}$.

En achetant l'hôtel d'Henri-Auguste de Loménie de Brienne, secrétaire d'État, le prince de Conti entra donc en possession d'une demeure qu'il n'éprouva pas le besoin de faire modifier et qu'il dut en conséquence juger conforme à son rang : il n'eut qu'à faire aménager une des pièces pour l'affecter à son conseil. En revanche, les dépendances qu'avait utilisées un simple particulier ne convenaient manifestement pas à un prince du sang et durent être largement complétées pour pouvoir abriter les différents services de sa maison et loger l'ensemble de son entourage. C'est cet impératif qui l'amena à supprimer le jardin de l'hôtel, qui était, il est vrai, moins essentiel ici que dans d'autres grandes demeures, la propriété tirant ses principaux agréments des vues dont on bénéficiait depuis ses appartements sur la Seine.

\section{Le grand hôtel de Conti sur le quai de Conti}

Après la mort en 1666 d'Armand de Bourbon, sa veuve conserva quelque temps leur hôtel parisien avant de l'échanger le 30 avril 1670 contre celui d'Henri de Guénégaud, ancien secrétaire d'État à la Maison du roi ${ }^{18}$. La princesse dut donner en soulte des terres, car la demeure qu'elle achetait était d'une valeur sensiblement supérieure à celle qu'elle abandonnait, et était même l'une des plus importantes habitations de la capitale. Elle aussi située au bord de la Seine (ill. 2), cette grande propriété était en effet organisée autour d'un imposant corps de logis construit dans les années 1570 et 1580 pour Louis de Gonzague, duc de Nevers, et transformé aux alentours de 1650 par François Mansart ${ }^{19}$. Celui-ci avait notamment ajouté un corps d'entrée, du côté de l'actuelle impasse de Conti, et avait fermé la cour au nord par une série de maisons locatives (ill. 3). Le même architecte avait également remanié l'intérieur du corps de logis principal, où il avait conçu pour

15. Arch. nat., $\mathrm{R}^{3} 110$, f. $355 \mathrm{v}-356$ et $\mathrm{R}^{3} 111$, f. $279 \mathrm{v}$.

16. Arch. nat., $R^{3} 108$, f. $450-450 v$.

17. Arch. nat., $\mathrm{R}^{3} 111$, f. 275 .

18. Arch. nat., Min. centr., ét. XCV, 35, 30 avril 1670.

19. Nous présentons ici un aperçu d'une étude plus complète sur cet hôtel : Étienne Faisant, "Un palais face au Louvre : de l'hôtel de Nevers à l'hôtel de Conti ", à paraître. 


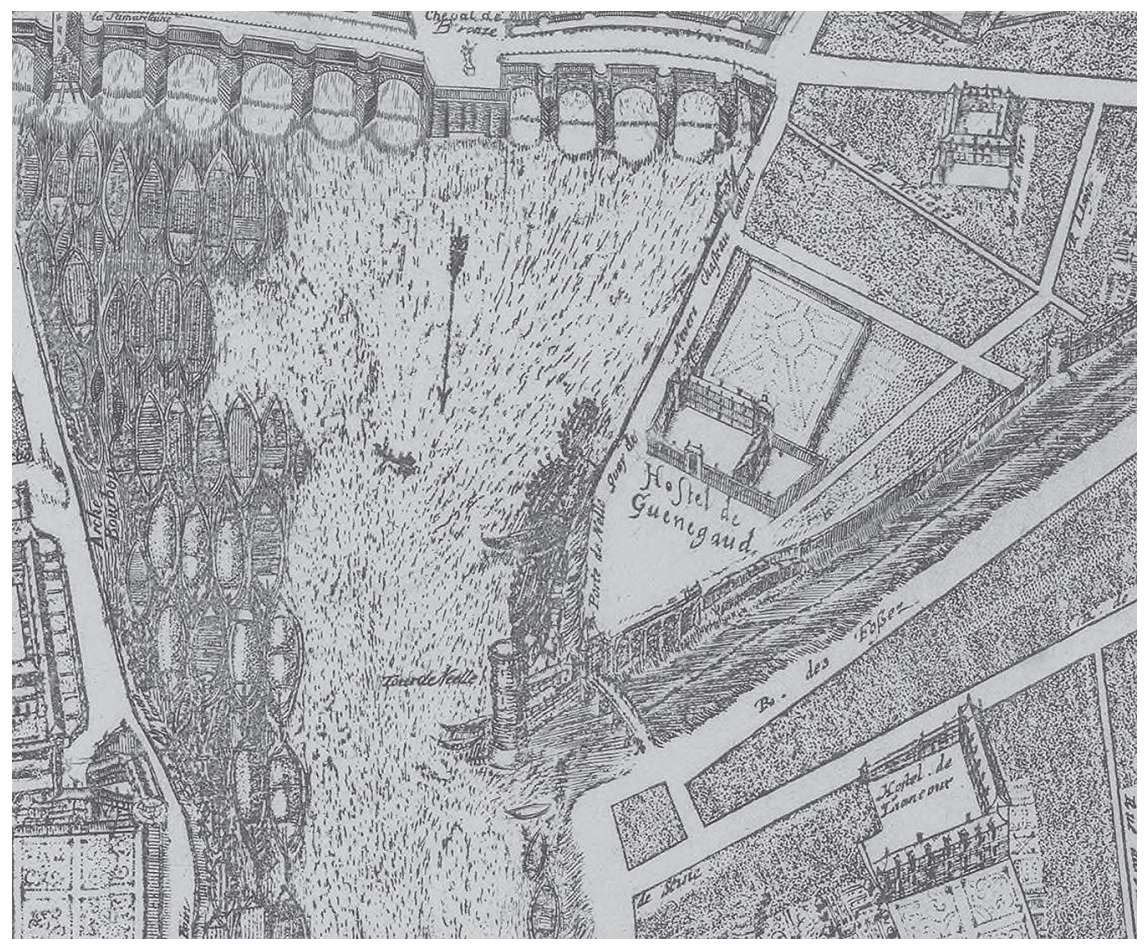

Ill. 2 : Détail de l'" hostel de Guénégaud ", futur hôtel de Conti, sur le plan de Paris par Jacques Gomboust, planche gravée, 1652. (c) Cl. BnF/Gallica.

Henri de Guénégaud et sa famille des appartements décrits avec admiration par Madeleine de Scudéry ${ }^{20}$.

Anne-Marie Martinozzi entreprit immédiatement de faire modifier sa nouvelle propriété pour l'adapter aux besoins de la famille princière. Outre la pose " au-dessus de la grande porte de l'hostel " d'« une ovale de marbre " portant l'inscription «Hôtel de Conty» commandée au sculpteur Martin Desjardins ${ }^{21}$ (ill. 4), elle fit notamment insérer dans le gros pavillon situé au nord un nouvel escalier à quatre noyaux et rampe de serrurerie, qui vint doubler celui préexistant situé au sud ${ }^{22}$ (ill. 5). Cet ajout réalisé sous la direction de l'architecte Siméon Lambert permit de diviser le premier étage en deux appartements différents. En empruntant l'ancien escalier méridional, on déboucha en effet dès lors à ce niveau dans un "grand appartement " qui, composé d'un "salon ", d'une antichambre, d'une chambre d'alcôve et d'un grand cabinet, était entièrement dévolu à l'apparat et à la réception. Au nord,

20. Madeleine de Scudéry, Clélie, histoire romaine, cinquième partie, Paris, Augustin Courbe, 1660, p. $478-480$.

21. Arch. nat., $\mathrm{R}^{3} 117$, f. 71 .

22. Arch. nat., $R^{3} 117$, f. 69-70. 


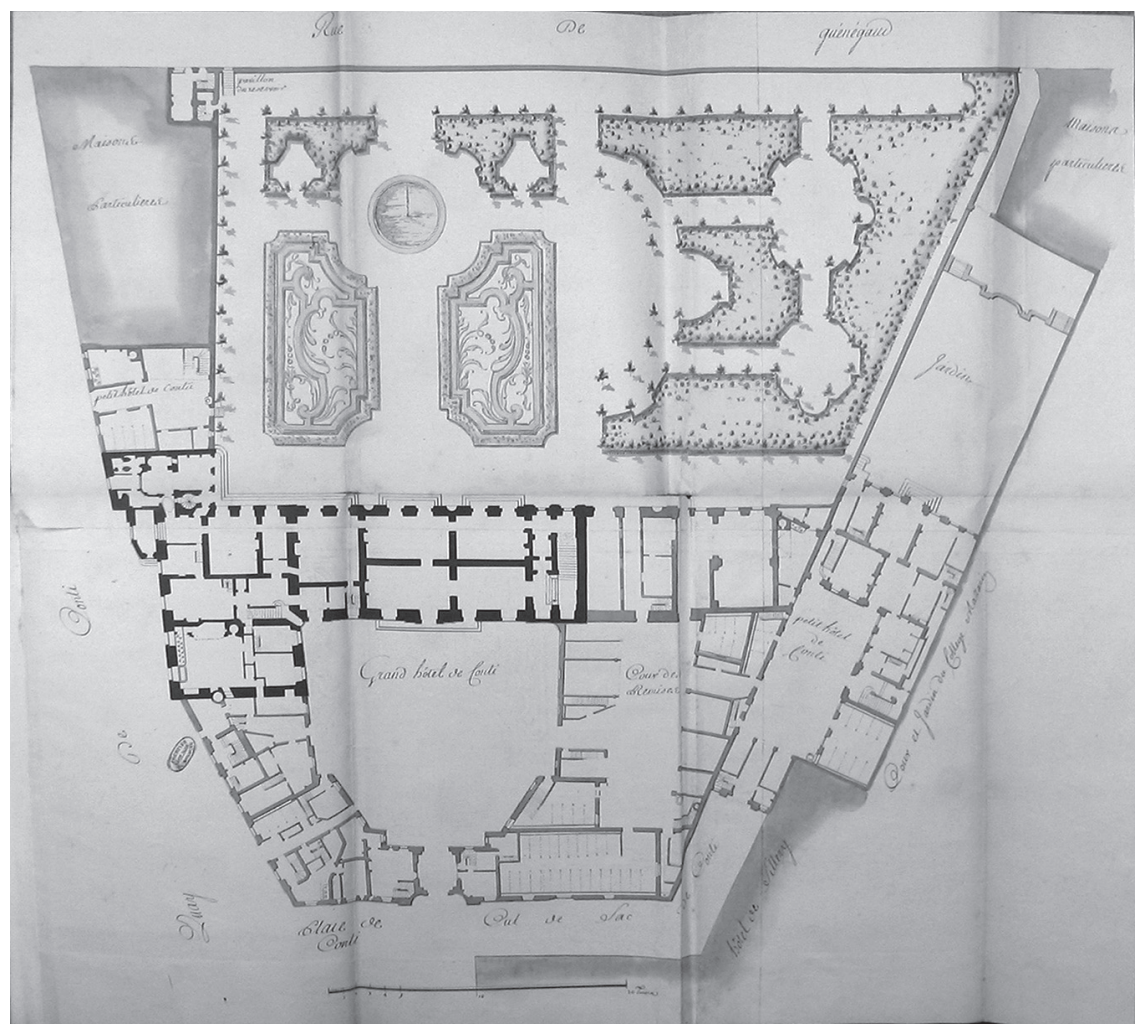

Ill. 3 : "Plan général de l'hôtel de Conti ", dessin à la plume et encre noire et grise rehaussé à l'aquarelle, 1750, Archives nationales, $\mathrm{R}^{3}$ 208. (C) Cl. Étienne Faisant.

le nouveau degré permettait quant à lui d'entrer dans une salle des gardes suivie par une antichambre ou salle à manger, par le vestibule de la chapelle et, enfin, par une chambre et une garde-robe, qui purent peut-être être utilisées par la princesse.

L'ajout du nouvel escalier présentait par ailleurs un autre intérêt, car il prenait place à côté des maisons longeant la cour, qu'Anne-Marie Martinozzi décida de ne plus louer : ces bâtiments furent utilisés pour abriter différents services et loger des serviteurs, la famille princière ayant à nouveau besoin de plus d'espaces que les précédents propriétaires des lieux, si riches fussent-ils. Le premier étage en fut en outre approprié pour former un logement de qualité supplémentaire, comprenant notamment une chambre d'alcôve. Cet appartement fut desservi par la même salle des gardes que celui sans doute occupé par la princesse.

Celle-ci s'attacha enfin à meubler au mieux sa nouvelle demeure. Elle avait acquis en même temps que l'édifice tous "les ajustemens, tableaux de chacuns dessus de porte et cheminée, comme aussy les autres choses servant à la décoration et embelissement dud. grand hostel et jardin d'iceluy " ainsi que pour vingt-deux 


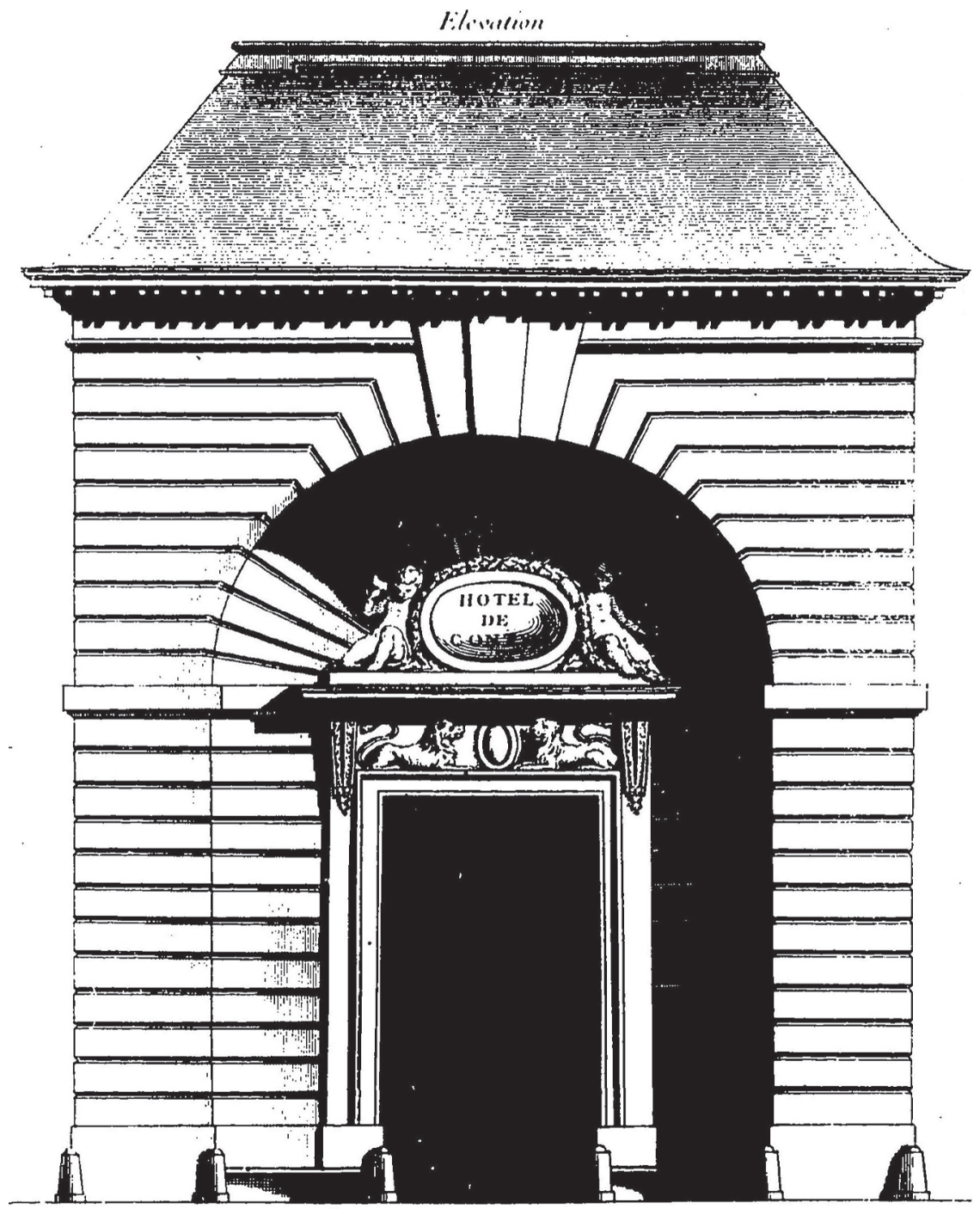

Ill. 4 : «Décoration extérieure de la porte d'entrée de l'hôtel de Conty située sur le quay de ce nom, près du collège Mazarin ", planche gravée, Jacques-François Blondel, Architecture françoise, Paris, 1752-1756. (C) $\mathrm{Cl} . \mathrm{BnF} /$ Gallica.

mille livres de meubles ${ }^{23}$, et elle disposait également de ceux qui avaient garni l'hôtel du quai Malaquais, mais elle jugea manifestement tout cela insuffisant pour sa nouvelle propriété. Elle acquit donc, entre autres, "une tanture de tapisserie antique 


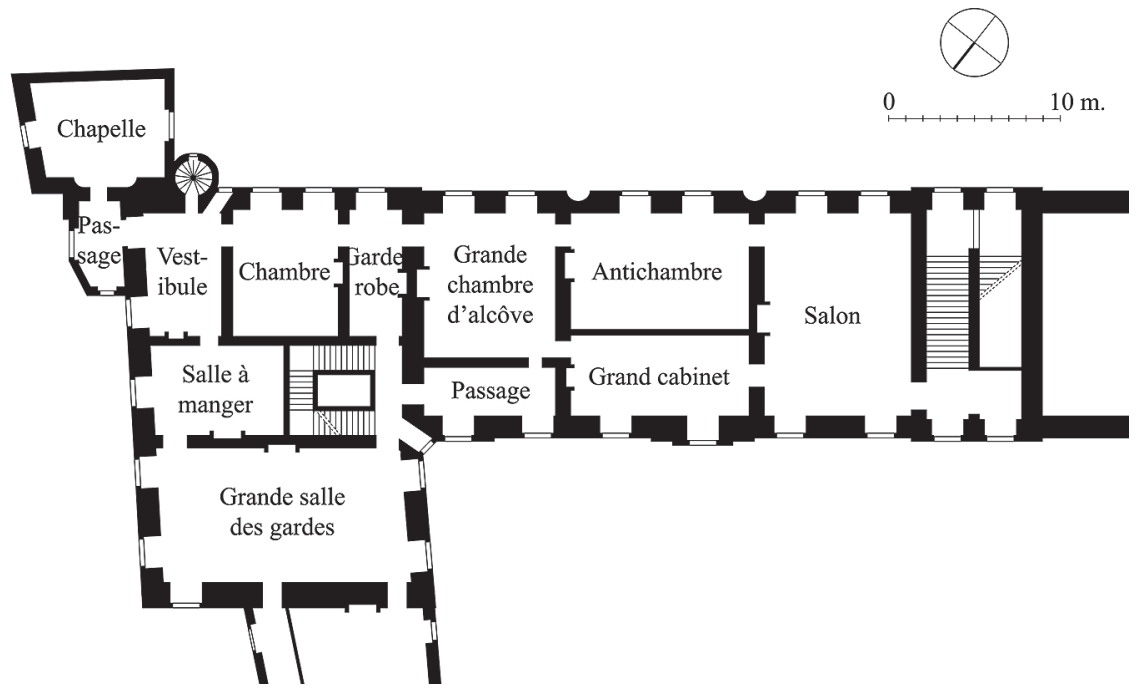

Ill. 5 : Plan restitué du premier étage de l'hôtel de Conti en 1683. C Dessin : Étienne Faisant.

d'aute lisse d'Angleterre représentant la bataille des Anglois contre les Bretons provenant de la succession de feu monsieur le duc d'Espernon » ${ }^{24}$. Elle commanda enfin au peintre "Laleman", sans doute Philippe Lallemand, un tableau pour orner la chapelle que François Mansart avait aménagé dans l'angle de l'hôtel ${ }^{25}$.

Après son décès, survenu en février 1672, ses deux fils mineurs furent élevés auprès du Dauphin, et l'on ne procéda donc alors pas à de nouvelles transformations majeures de leur résidence parisienne. En 1678-1679, les chambres du nouveau prince de Conti et de son cadet, le prince de La Roche-sur-Yon, furent néanmoins entièrement redécorées et dotées de nouvelles peintures dues à Jean Lemoyne et à René-Antoine Houasse ${ }^{26}$.

Le mariage, le 16 janvier 1680, de Louis-Armand de Bourbon, prince de Conti (1661-1685), avec Marie-Anne de Bourbon, fille de Louis XIV et de la duchesse de La Vallière, imposa toutefois de répartir différemment les espaces: le jeune couple conserva l'ensemble de l'hôtel, tandis que le prince de La Roche-sur-Yon déménagea dans un petit hôtel voisin, construit par Jules Hardouin-Mansart en 1669 pour Henri de Guénégaud et acheté dix ans plus tard par les tuteurs des Conti $^{27}$ (ill. 6). La chambre de l'ancien appartement de la mère du prince au pre-

24. Arch. nat., $\mathrm{R}^{3} 117$, f. $38 \mathrm{v}$.

25. Arch. nat., $R^{3} 117$, f. 36-36v.

26. Arch. nat., $\mathrm{R}^{3} 132$, f. $224 \mathrm{v}-230 \mathrm{v}$ et $\mathrm{R}^{3} 133$, f. $195 \mathrm{v}-198$.

27. Bertrand Jestaz, "La première œuvre connue de Jules Hardouin-Mansart : le petit hôtel de Guénégaud, dit de Conti ", Bulletin monumental, Paris, Société française d'archéologie, t. 158-3, 2000, p. 217-237 ; Arch. nat., Min. centr., ét. XCII, 226, 25 mai 1579 et R $\mathrm{R}^{3}$ 61, $1^{\text {er }}$ avril et 8 juillet 1680 ; Germain Brice, Description nouvelle de ce qu'il y a de plus remarquable dans la ville de Paris, Paris, Nicolas Le Gras, 1684, t. I, p. 219. 


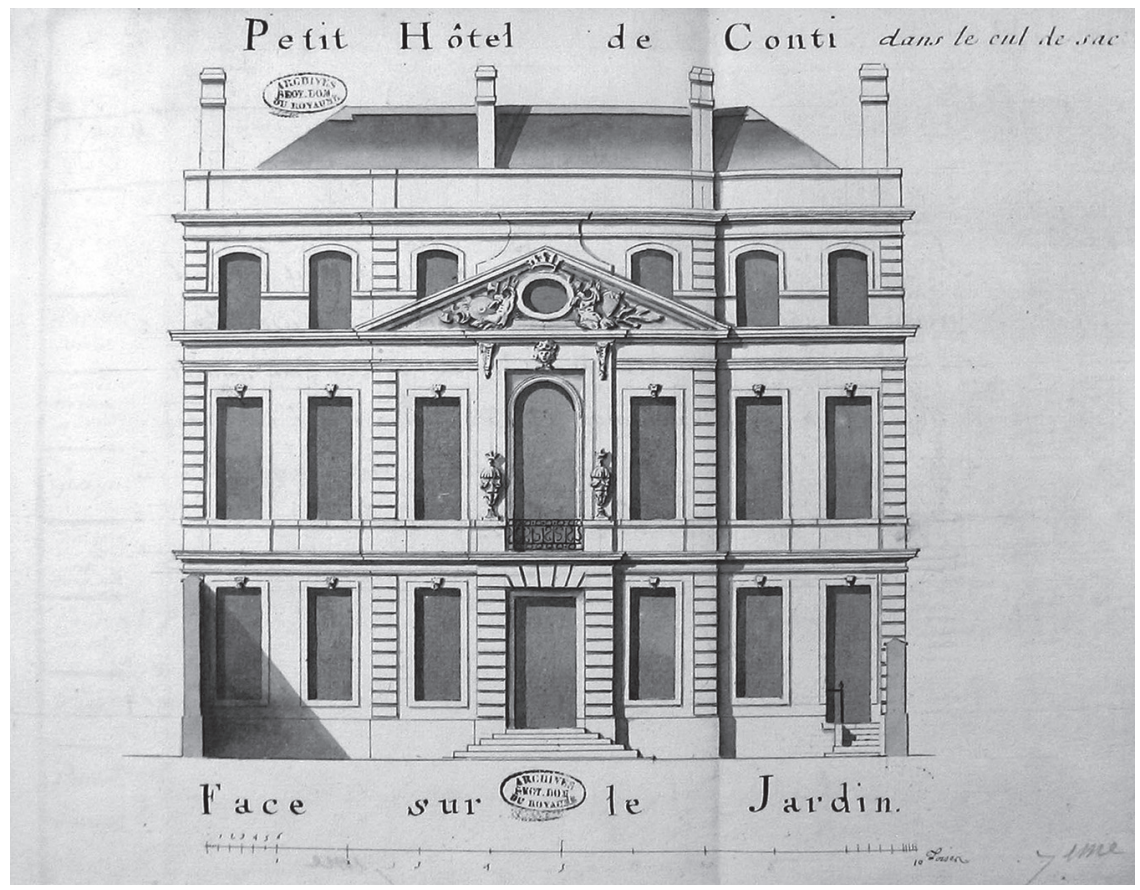

Ill. 6 : "Petit hôtel de Conti, face sur le jardin ", dessin à la plume et encre noire et grise rehaussé à l'aquarelle, 1750, Archives nationales, $\mathrm{R}^{3}$ 208. (C) Cl. Étienne Faisant.

mier étage fut alors remise en état pour la nouvelle princesse ${ }^{28}$, et, sous la direction de l'architecte Daniel Gittard, de nouveaux travaux de maçonnerie, de charpenterie, de couverture, de peintures, de dorures et de marbrerie furent réalisés pour aménager un appartement des bains disposé du côté du quai, dans l'angle du jardin ${ }^{29}$.

La mort prématurée du prince de Conti, le 9 novembre 1685, entraîna une nouvelle redistribution des lieux. L'hôtel fut en effet réinvesti par son cadet FrançoisLouis (1664-1709), qui quitta le petit hôtel pour reprendre possession du grand. Il en occupa personnellement le rez-de-chaussée, où il fit poser de nouveaux décors : l'un des cabinets fut doté en 1687-1688 d'une nouvelle cheminée et, au-dessus de murs garnis de glaces peintes, d'un nouveau plafond dû à Jean Jouvenet représentant Zéphire et Flore ${ }^{30}$. Dans le même temps, le prince commanda à Antoine Coysevox un buste en bronze de son oncle le Grand Condé ${ }^{31}$. Réalisé "sous la

28. Arch. nat., $\mathrm{R}^{3} 137$, f. $34 \mathrm{v}-35$.

29. Arch. nat., $\mathrm{R}^{3} 137$, f. $37-37 \mathrm{v}, 39$ et $70-73$.

30. Arch. nat., AB XIX 3295, dossier 3, mémoire de Jean Duchesnoy, février 1688 ; Antoine-Joseph Dezallier d'Argenville, Abrégé de la vie des plus fameux peintres, Paris, De Bure l'aîné, 1745, t. II, p. 355 ; Antoine Schnapper, Jean Jouvenet, 1644-1717, et la peinture d'histoire à Paris, nouvelle édition complétée par Christine Gouzi, Paris, Arthéna, 2010, p. 90, 213 et 331.

31. Ce buste est aujourd'hui au Louvre (M.R. 3343). 
conduitte de M. Mansart, premier architech de Sa Majesté ", cet hommage à son tuteur et protecteur décédé en décembre 1686 et dont il devait épouser dans les mois suivants l'une des petites-filles fut posé au début de 1688 dans la première salle de l'appartement ${ }^{32}$.

L'installation au premier étage de la nouvelle princesse de Conti ne semble pas avoir nécessité de nouveaux travaux, ceux réalisés pour sa belle-sœur huit ans auparavant ayant dû sembler suffisants. Le prince commanda en revanche en 1699 à Jouvenet un nouveau plafond pour son propre appartement, sans doute pour sa chambre : le peintre y représenta «la Renommée, la Gloire, l'Abondance avec un génie tenant une couronne ${ }^{33}$. En 1706, François-Louis de Bourbon fit enfin détruire la chapelle de François Mansart pour faire aménager à son emplacement, au premier étage du pavillon élevé à l'angle du jardin, "un grand cabinet dont les vues règnent sur la rivière, embelli d'une excellente menuiserie dorée " ${ }^{34}$. Une terrasse fut également aménagée à côté et l'appartement des bains fut remanié pour s'étendre dans l'ensemble du rez-de-chaussée de ce pavillon ${ }^{35}$. Le prince mourut trois ans plus tard, en 1709. Il habitait alors toujours avec sa femme le corps principal de l'hôtel, tandis que leurs enfants étaient répartis entre le petit hôtel et le premier étage de l'aile gauche, entre la cour et le quai ${ }^{36}$.

\section{L'abandon du grand hôtel de Conti}

Louis-Armand, comte de La Marche (1695-1727), succéda alors à son père comme prince de Conti et hérita entre autres de son hôtel parisien, où sa mère, après avoir envisagé de s'établir rue de Lille ${ }^{37}$, décida d'habiter avec lui. Elle demanda toutefois à Pierre-Alexis Delamair de repenser entièrement cette demeure, pour laquelle l'architecte proposa un projet rappelant fortement ce qu'il avait fait quelques années plus tôt à l'hôtel de Soubise : il envisagea de retourner la propriété en aménageant à

32. Château de Chantilly, archives, O, t. VIII, p. 265-266, publié par Louis Courajod, "Portrait du grand Condé au musée du Louvre, par Antoine Coyzevox ", La Chronique des arts et de la curiosité, Paris, Gazette des Beaux-Arts, 3 mars 1877, p. 84-85.

33. Théophile Lhuillier, "Note relative à Jean Jouvenet et à ses filles ", Réunion des Société des Beaux-Arts des départements, Paris, Plon, Nourrit et C $C^{\text {ie }}, 1889$, p. 447-453, p. 449 ; Antoine-Joseph Dezallier d'Argenville, Abrégé..., op. cit., t. II, p. 355.

34. Germain Brice, Description nouvelle de la ville de Paris, $5^{\mathrm{e}}$ édition, Paris, Nicolas Le Gras, 1706, t. IV, p. 380 ; $6^{\text {e }}$ édition, Paris, François Fournier, 1713, t. III, p. 199.

35. En 1749, prenaient place au rez-de-chaussée un "cabinet des bains" et une "garde-robe des bains", et à l'étage le "sallon doré" (Arch. nat., $\mathrm{R}^{3}$ 208). Ces dispositions étaient déjà en place en 1709 puisque l'inventaire dressé cette année-là mentionne "le cabinet aux bains ayant veue sur le jardin " et la "petitte garderobe ayant veue sur l'eau " (Arch. nat., Min. centr., ét. XCII, 389, 25 mars 1709).

36. Arch. nat., Min. centr., ét. XCII, 389, 25 mars 1709.

37. Philippe Courcillon, marquis de Dangeau, Journal du marquis de Dangeau, éd. Eudore Soulié, Louis Dussieux, Charles-Philippe de Chennevières-Pointel, Paul Mantz et Anatole de Montaiglon, Paris, Firmin Didot, 1854-1860, t. 17, p. 410-411, 29 novembre 1718. L'hôtel dont elle avait entrepris la construction rue de Lille fut vendu en 1718 à son beau-frère et à sa sœur le duc et la duchesse du Maine. 


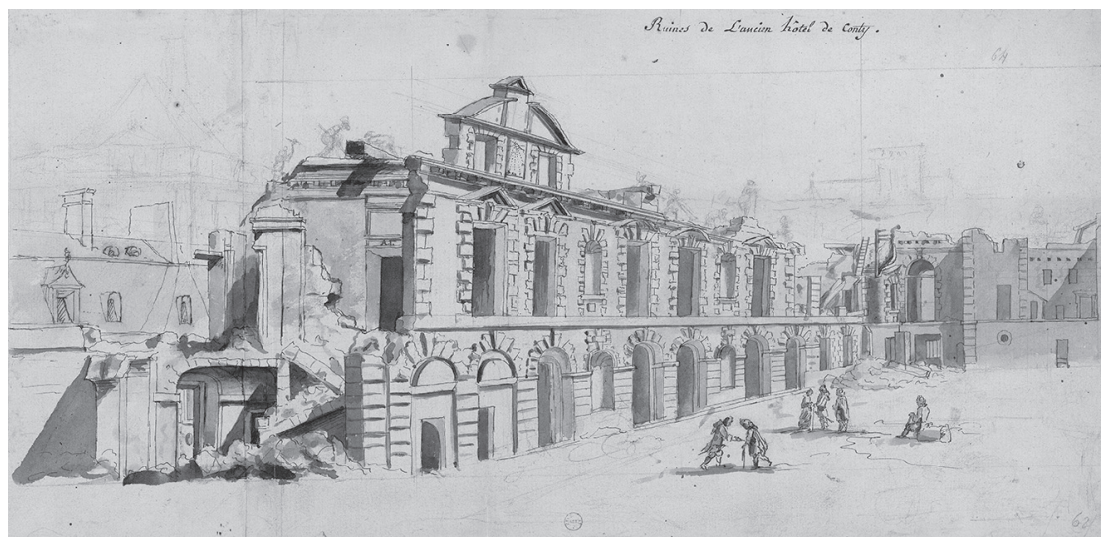

Ill. 7 : «Ruines de l'ancien hôtel de Conty ", dessin à la mine de plomb, plume et lavis à l'encre brune, vers 1768, BnF, Estampes, Rés. Fol. Ve 53(F). (C) Cl. BnF/Gallica.

l'emplacement du jardin une cour bordée de colonnades portant des terrasses et en créant à la place de l'ancienne cour un nouveau jardin d'où l'on aurait joui de vues sur " la rivière, les Thuilleries et la campagne " ${ }^{38}$.

Ce projet ne fut toutefois pas mis en œuvre, peut-être parce que, comme le pensa Delamair, ses rivaux convainquirent le prince de Conti de faire plutôt travailler dans son château de L'Isle-Adam ${ }^{39}$, mais plus probablement parce que la princesse douairière, qui avait commandé ce projet, changea une fois encore d'avis et décida à nouveau d'avoir son propre hôtel ${ }^{40}$. Son fils se limita en tout cas dans sa demeure parisienne à des travaux plus réduits, qu'il semble avoir confiés à Germain Boffrand ${ }^{41}$. C'est peut-être au cours de cette campagne que la salle du rez-de-chaussée fut divisée par un mur pour former deux antichambres successives, état décrit dans l'inventaire dressé après la mort du prince en $1727^{42}$.

Si son propre fils Louis-François de Bourbon (1717-1776) fit à nouveau mener en 1742 de petits travaux dans l'hôtel ${ }^{43}$, il chercha dès 1748 à le vendre à la Ville de Paris afin que celle-ci aménage à son emplacement la nouvelle place royale alors en projet ${ }^{44}$. La transaction fut conclue en décembre 1751 moyennant un million

38. Bibliothèque de l'Arsenal, Ms 3054 (Pierre-Alexis Delamair, La pure vérité, 1737), p. 108.

39. Bibliothèque de l'Arsenal, Ms 3054, p. 114.

40. Arch. nat., Min. centr., ét. XCII, 469, 29 février 1732.

41. Bruno Pons, "Germain Boffrand et le décor intérieur ", Michel Gallet et Jörg Garms (dir.), Germain Boffrand, 1667-1754, l'aventure d'un architecte indépendant, Paris, Herscher, 1986, p. 187-252, p. $214-215$ et 250 , n. 38 .

42. Arch. nat., Min. centr., ét. XCII, 450, 23 mai 1727 ; Arch. nat., R 208.

43. Évelyne Olivier-Valengrin, "Le château... ", op. cit., p. 124, n. 35.

44. René-Louis de Voyer d'Argenson, Journal et mémoires du marquis d'Argenson, éd. Edmé-Jacques-Benoît Rathery, Paris, veuve Renouard, 1859-1867, t. V, p. 258 : « M. de Bernage, prévôt des marchands, m'a conté hier ce qui suit sur ces monuments dont il est grande question [...] : c'est M. le prince de Conti qui a mis tout cela en branle, pour bien vendre son hôtel à la ville de Paris " (octobre 1748). 
six cents mille livres ${ }^{45}$, et, s'il fut comme on le sait finalement décidé de créer ailleurs la nouvelle place Louis XV, le grand hôtel de Conti fut rasé en 1768 pour laisser place à l'hôtel de la Monnaie (ill. 7).

Après s'être ainsi séparé de ce qui avait été durant huit décennies la demeure des princes de Conti à Paris, Louis-François de Bourbon s'installa un temps dans l'hôtel dont il avait hérité l'année précédente de sa tante Louise-Adélaïde de Bourbon, Mademoiselle de La Roche-sur-Yon. Celui-ci n'était autre que l'ancien hôtel de Conti sur le quai Malaquais qui, devenu hôtel de Guénégaud, de Créquy puis de Lauzun, avait été dans l'intervalle sensiblement remanié. Le prince semble y avoir habité jusqu'en 1757 avant de déménager dans l'hôtel du Temple, dont il avait la jouissance depuis sa nomination en 1749 comme Grand Prieur de France et où il avait fait mener entre-temps d'importants travaux ${ }^{46}$. Son fils Louis-François-Joseph (1734-1814), alors connu sous le nom de comte de La Marche, habita pour sa part l'ancien hôtel de Rothelin, rue de Grenelle, hérité en 1758 de sa grand-tante Mademoiselle de Charolais. Il ne paraît pas avoir fait entreprendre d'importantes modifications dans cette demeure ${ }^{47}$, où il resta jusqu'à la Révolution et qui, le prince étant mort en exil en 1814, fut le dernier hôtel de Conti à Paris.

Étienne FAISANT Post-doctorant, labex EHNE, Sorbonne Université

45. Arch. nat., P 2031.

46. Frédéric Dassas, "Les résidences parisiennes des princes de Conti ", dans Les trésors des princes de Bourbon-Conti, catalogue de l'exposition tenue en 2000 au musée Louis-Senlecq à L'Isle-Adam, p. 97-101, ici p. 98-99. Les travaux furent terminés vers 1757, date probable du déménagement du prince, ce qui laisse supposer qu'il s'était installé quai Malaquais dans l'attente de leur achèvement.

47. François Béchade, L'hôtel de Rothelin puis de Charolais, XVIII $-X I X{ }^{e}$ siècle, mémoire d'étude sous la direction d'Alexandre Gady, École du Louvre, 2016, notamment p. 96-98. 